\title{
ULTRASONICALLY INDUCED MORPHOLOGICAL CHANGES IN THE MAMMALIAN NEONATAL SPINAL CORD
}

\author{
M. J. Borrelli, $\dagger$ L. A. Frizzell and F. DunN $\ddagger$ \\ Bioacoustics Research Laboratory, University of Illinois, 1406 West Green Street, Urbana, IL 61801, U.S.A. \\ (Received 12 August 1985 and in final form 4 December 1985)
}

\begin{abstract}
Light and electron microscopy were employed to examine ultrasonically irradiated murine neonate spinal cords. Spinal cord damage was always associated with functional changes, i.e. hind limb paralysis, and was never apparent in initially paralyzed specimens which had recovered function. Damage occurred preferentially at the periphery of the spinal cord and on the ventral side, though the ultrasound was incident on the dorsal side. The distribution of the damage suggests spinal column involvement and nonthermal effects that are enhanced by temperature elevation.
\end{abstract}

Key Words: Acoustics, Ultrasonics, Ultrasonic tissue damage, Ultrasonic toxicity, Spinal cord, Hind limb paralysis.

\section{INTRODUCTION}

The central nervous system has often served as a useful organ for the investigation of the biological effects of ultrasound. Studies have involved the examination of functional [Fry and Dunn, 1956] and structural [Fry et al., 1970] changes associated with ultrasound exposure. Hind limb paralysis in neonatal mice has been employed as an endpoint for assessing ultrasonically induced spinal cord damage [Fry and Dunn, 1956; Dunn, 1958]. The neonatal mouse is a suitable model for investigating mechanisms of damage as it is an essentially poikilothermic animal allowing for broad temperature variations. It is also small so that exposure chambers can be constructed readily to permit hyperbaric conditions. For selected values of the acoustic intensity of exposure, the base temperature of the poikilothermic animals, and the hydrostatic pressure, it has been observed that a sigmoid shaped curve describes the relation of the fraction of the specimens paralyzed to the reciprocal of the exposure duration. This has provided for a threshold range to be defined as the exposure range yielding $10-90 \%$ of the animals paralyzed. The ultrasonically induced temperature rises, determined by spinal cord-embedded thermo-

$\dagger$ Present address: Department of Radiation Oncology, Temple University School of Medicine, 3401 Broad St., Philadelphia, PA 19140, U.S.A.

$\ddagger$ Author to whom correspondence should be addressed. couples [Fry and Dunn, 1956; Dunn, 1958] did not raise the cord temperature of the cooled animals to nearly the normal temperature of the adult animals. Thus, it was considered that the changes in spinal cord function and morphology were not produced by thermal means alone.

Frizzell et al. [1983] have observed that initially paralyzed mice, under certain specified conditions, have recovered hind limb function within $15-20 \mathrm{~min}$ post irradiation. They also reported an increase in the threshold exposure duration required for both irreversible and reversible paralysis at the hyperbaric pressure of $16 \mathrm{~atm}$, when the acoustic intensity was 289 $\mathrm{W} / \mathrm{cm}^{2}$. No such increase was found for an acoustic intensity of $144 \mathrm{~W} / \mathrm{cm}^{2}$ and less. Also, the hyperbaric pressure reduced the attending subharmonic frequencies, providing additional evidence that acoustically induced cavitation was present.

These studies were undertaken largely to establish ultrasonic thresholds with little attention devoted to investigating associated changes in tissue morphology. Fry and Dunn [1956] and Dunn [1958] did observe cell lysis and necrosis, and Taylor [1970], using adult animals, observed hemorrhaging. As these studies employed light microscopy to observe morphologic changes, subtle tissue alteration may have gone unobserved. This is supported by the fact that altered tissue morphology observable with light microscopy did not appear until approximately $15 \mathrm{~min}$ post irradiation, 
while paralysis always occurred immediately [Dunn, 1958].

In the present study both light and electron microscopy are employed to examine ultrasonically irradiated murine neonate spinal cords to determine: (1) the relation of altered tissue morphology to irreversible and reversible hind limb paralysis; (2) the nature and distribution of the tissue damage associated with sub-, supra-, and threshold exposures; (3) the development of tissue damage as a function of post irradiation time; (4) the effects of hyperbaric pressure on the nature of the tissue damage; and (5) the physical mechanism(s) by which the ultrasound induced the observed changes.

\section{MATERIALS AND METHODS}

The neonatal mouse irradiation procedure and equipment have been described elsewhere [Fry and Dunn, 1956; Frizzell et al., 1983]. Briefly, ICR [Hap: (ICR)BR Harland Industries Inc., Indianapolis, IN] mouse neonates, harvested in the laboratory approximately $24 \mathrm{~h}$ postnatal and weighing from $1.5-1.8 \mathrm{~g}$, were anesthetized, just prior to employment, by cooling on ice until dormant. Each specimen was positioned in a holding device such that the third lumbar vertebra was centered in the unfocused acoustic field. The irradiation chamber and transducer system were identical to that used by Frizzell et al. [1983], and all irradiations were performed at the ambient temperature of $10^{\circ} \mathrm{C}$. Degassed Ringer's solution filled the irradiation chamber and served as the acoustical coupling medium. The animal's head was sealed, using petroleum jelly, into a portion of the holder that was connected, via a rubber tube, to the air above the saline surface, thus permitting free breathing at all times.

Approximately 4 min was required for the mouse to reach temperature equilibrium in the $10^{\circ} \mathrm{C}$ irradiation chamber, at which time it was exposed to the 1 $\mathrm{MHz}$ unfocused continuous wave ultrasound, incident on its dorsal side. At the specimen position within the sound field, viz. $10 \mathrm{~cm}$ from the transducer face, the transverse width of the acoustic beam, to points $95 \%$ of peak intensity, was approximately $3 \mathrm{~mm}$, ensuring a spatial intensity variation of less than $5 \%$ over the third lumbar region of the spinal cord. The sound field distribution was determined using a thermocouple probe, prior to the specimen exposures [Fry and Dunn, 1956].

The specimens were irradiated at ultrasonic intensities between 40 and $289 \mathrm{~W} / \mathrm{cm}^{2}$, for exposure times chosen to cover the threshold range associated with each acoustic intensity. Exposure durations corresponded to $t_{10}, t_{50}$, and $t_{90}$ values reported by Frizzell et al. [1983], i.e. the exposure duration which produced paralysis in 10,50 , and $90 \%$, respectively, of the irradiated specimens. During 20 irradiations, receiving transduccrs were used to record the frequency spectra of the sound waves in the irradiation chamber. A 0.5 $\mathrm{MHz}$ narrowband acoustic monitor recorded the subharmonic frequencies and a broadband detector was used for the higher harmonics and anharmonic signals. Most of the exposures were carried out at atmospheric pressure, with 35 performed at the increased hydrostatic pressure of 16 atm [Frizzell et al., 1983]. Sham exposures were treated identically except that zero intensity ultrasound was delivered.

Immediately following the irradiation procedure, the animal, still in the specimen holder, was placed into a $37^{\circ} \mathrm{C}$ water bath for $4 \mathrm{~min}$ to facilitate its recovery from the anesthesia. The specimen was then removed from the holder and tested for hind limb paralysis by applying stimulating pinches lightly on each hind limb and the tail, in sequence. Sham and control (control specimens received no pretreatment) animals responded to the stimulation by moving both the hind and forelimbs and frequently emitting a vocal squeak. In order to eliminate confusion between back movement and hind limb movement, each animal was held such that the back was immobilized, but the hind limbs were unrestricted. Records were kept on the animal's ability to sense the stimulating pinch, as indicated by the vocal squeak and/or forelimb movement. An identical test was performed on the forelimbs to (a) ensure that they were mobile, indicating recovery from the anesthesia; and (b) eliminate the possibility that hind limb motion failed to occur because the animal could not sense the hind limb stimulations, as opposed to being paralyzed. Sham and control specimens responded to forelimb stimulus as they did to hind limb stimulus, hence failure of the test specimen to move the hind limbs following forelimb stimulation was further confirmation of hind limb paralysis. A post irradiation time of at least $15 \mathrm{~min}$ was required to determine unequivocally whether the specimen was irreversibly or reversibly paralyzed [Frizzell et al., 1983].

A second test for paralysis and stimulation sensation was performed prior to sacrifice, following post irradiation times of 4 min to $4 \mathrm{~h}$. After testing, the animal was decapitated and bisected, either inferior or superior to the second lumbar vertebra, using a tissue slicer [Duffy and Tyler, 1975]. The lower portion of the specimen was placed immediately into an icecooled petri dish filled with fixative consisting of $2.5 \%$ gluteraldehyde and $2.0 \%$ paraformaldehyde in $0.1 \mathrm{M}$ cacodylate buffer at a $\mathrm{pH}$ of 7.2. Although the lower temperature slowed fixation, it minimized cellular degeneration, resulting in tissue preservation superior to that attained at room temperature. The specimen was 
swirled in the fixative for $30-45 \mathrm{~s}$ to remove blood and then transferred to another cooled petri dish containing fresh fixative. Using a dissecting microscope to observe the specimen, a sharp razor blade was employed to isolate the irradiated portion of the spinal cord by cutting through the spinal column inferior to either the third or fourth lumbar vertebra. Microdissection procedures were used to remove the spinal cord segment from the spinal column, and the spinal cord segment was stored in a labeled vial filled with fixative. The entire sacrifice and dissection procedure was performed in less than $4 \mathrm{~min}$. Fifty-four specimens, more than $25 \%$ of the animals, were prepared for histological examination blind, viz. the individual conducting the tissue preparation and the microscopic viewing was possessed of no information of the ultrasonic exposure conditions. Control and sham-irradiated animals were included in the blind phase of the study.

Labeled vials were stored an additional $24 \mathrm{~h}$ at $4^{\circ} \mathrm{C}$. The spinal cord segments were cut transversely into $0.25 \mathrm{~mm}$ thick serial sections using the tissue slicer. The relative position of each section was determined by its cross-sectional diameter, as the spinal cord narrows sharply between the second and fourth lumbar vertebrae [Rugh, 1968]. After preparation for electron microscopy [Borrelli et al., 1981], $1 \mu \mathrm{m}$ thick sections were cut from the specimen block, affixed to glass slides, and stained with $0.5 \%$ toluidine blue for light microscopic observation. Selected regions of the embedded tissue were thin sectioned (500-700 $\AA$ thick) and examined with a JEOL JEM $100 \mathrm{C}$ transmission electron microscope using an accelerating voltage of $80 \mathrm{kV}$.

\section{RESULTS}

Spinal cords from all specimens exhibiting the phenomenon of reversible paralysis revealed morphology indistinguishable from that observed in the control and sham irradiated specimens. As no exposure conditions yielded $100 \%$ reversible paralysis, the animals had to recover hind limb function before being identified as such. Exposure conditions which yielded a high percentage of reversibly paralyzed mice also exhibited a lesser incidence of abnormal morphology in paralyzed animals sacrificed immediately after irradiation, and at later post irradiation times less than the mean post irradiation lime for return of hind limb function to specimens confirmed as reversibly paralyzed. This suggests that at no time prior to hind limb function recovery did reversibly paralyzed mice experience observable ultrasonically induced tissue damage.

Spinal cord tissue taken from mice exhibiting irreversible paralysis always exhibited some degree of altered tissue morphology. Both the degree and distri- bution of tissue damage in these spinal cord specimens depended upon the acoustic intensity, the exposure time, and the time post irradiation at which the specimen was sacrificed. It is to be noted that spinal cords from identically treated animals did not necessarily exhibit identical degrees of tissue damage. As there was a variation in the functional effects exhibited in a population of identically treated specimens, there also was variation in the degree of observed tissue damage ranging from no effect to, in some instances, macroscopic tissue disruption visible with the naked eye.

The nature of the observed altered morphology was similar for all exposure intensities, with differences appearing in distribution throughout the cord. For the purpose of describing the distribution of the tissue damage, the spinal cord cross section is divided into eight regions, as illustrated in Fig. 1. Regions 1-4 along the spinal cord periphery represent that tissue which develops into the white matter. The most commonly observed structures herein were the nonmyelinated axons, usually seen in cross section (Fig. 2). Both mitochondria and the microtubules were observed within these axons. Other structures within regions 1-4 included some cell bodies, and sections through cellular processes, viz. dendrites and synapses. Figure 3 shows the synapse morphology in greater detail. The presynaptic element is filled with membrane-bound synaptic vesicles. The boundary between it and the postsynaptic element is indicated by an electron dense region, the junctional density. In this tissue it was generally not possible to determine whether a nonsynaptic process was a dendrite or a portion of a glial cell. For this reason, they are grouped together and referred to as dendritelike processes (Fig. 2). Regions 5-8 contained more cellular bodies (both neurons and glial), more dendritelike processes, and a reduced number of nonmyelinated axons (Fig. 4).

A. Altered morphology arising from exposure to 289 $W / \mathrm{cm}^{2}$

The minimum tissue damage associated with hind limb paralysis was restricted to region 1 on the ventral side of the cord, and required the electron microscope for detection. Most of the dendritelike processes appeared slightly swollen and less electron dense than in the control and sham tissue, and often contained amorphous, membranous debris (Fig. 5). Many synapses also appcarcd swollen, had less electron dense junctional densities, contained membranous debris, and exhibited a reduced number of synaptic vesicles [Fig. 5(a)]. Most of the remaining vesicles were aggregated at the junctional density. The mitochondria exhibited varying degrees of reduced electron density within their matrices. Most had a mild overall density 

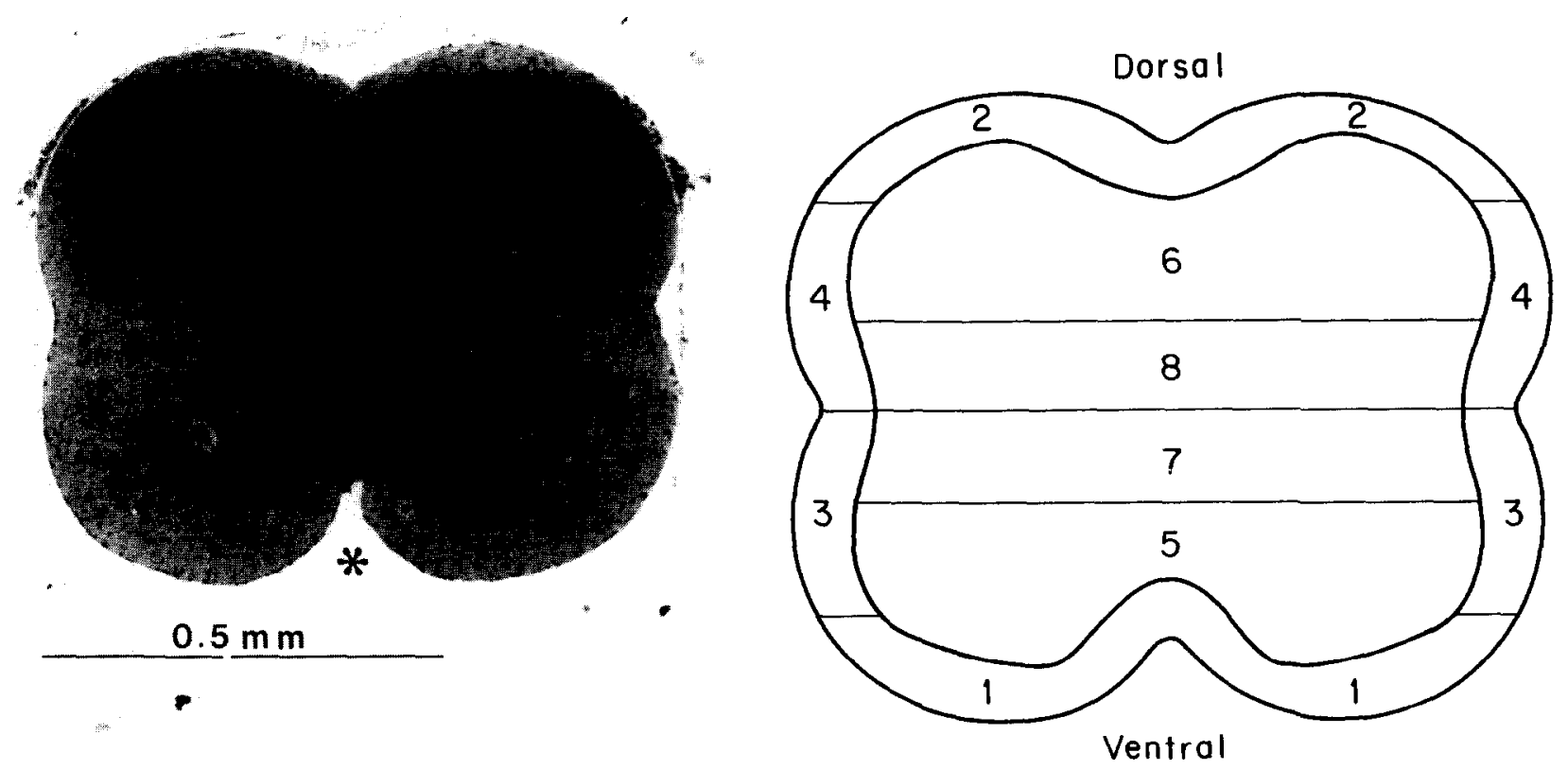

(b)

Fig. 1. (a) Light micrograph of normal third lumbar spinal cord cross section. The asterisk indicates the ventral half of the section. (b) Regions of the spinal cord cross section.

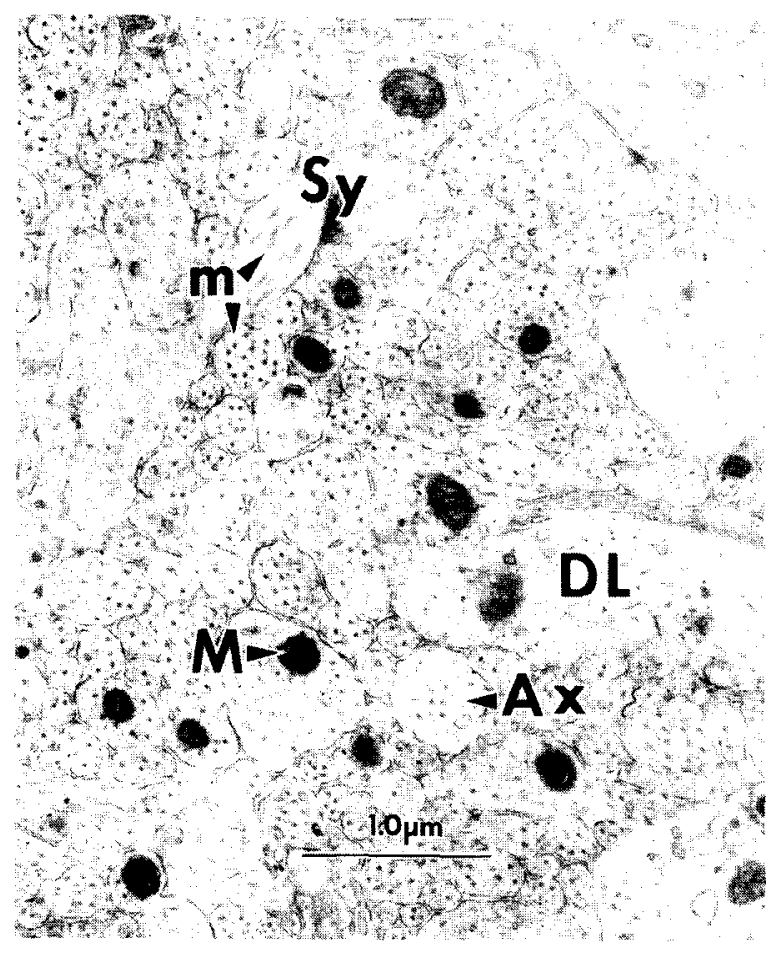

Fig. 2. Normal ultrastructure of peripheral spinal cord tissue. Dendritelike processes DL, nonmyelinated axons Ax, and Synapses Sy were the structures most commonly observed. Mitochondria $\mathrm{M}$ and microtubules $\mathrm{m}$ are also indicated. reduction, some contained transparent zones [arrowheads in Fig. 5(a)], while others exhibited a completely electron lucent matrix [Fig. 5(b)]. In the latter case, the mitochondria appeared distinctly swollen with their cristae displaced towards the periphery. Both mitochondrial membranes remained intact despite the apparent swelling. The least affected structures were the nonmyelinated axons, particularly those with the smaller diameters. Only a small percentage exhibited lower electron densities and other signs of swelling. Damage of the degree described above (and illustrated in Fig. 5) was characteristic of that observed within the spinal cords of mice paralyzed with the $t_{10}$ exposure duration and sacrificed within 4-30 min post irradiation.

Spinal cords from mice paralyzed with the $t_{50}$ and $t_{90}$ exposure durations exhibited tissue damage ranging in degrees from that described above to damage observable with the naked eye. In over $60 \%$ of these animals, tissue damage extended into spinal cord region 3 with a smaller percentage also having damage in region 5. The lowest degree of damage in these regions was similar to that described above for region 1 . Sections that sustained slightly more damage exhibited altered morphology within the cell bodies, with the neurons appearing to be more sensitive to the exposure than the glial cells. Figure 6 shows a neuron in which 


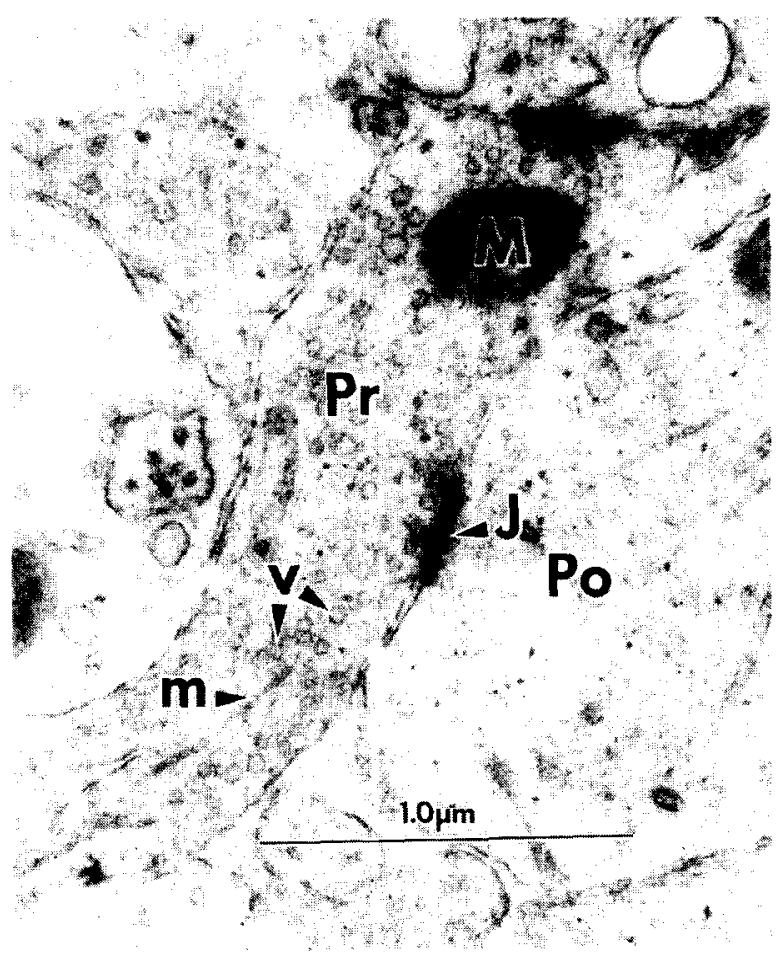

Fig. 3. Synapse morphology. The electron dense junctional density. J marks the tight junction between the presynaptic Pr and postsynaptic Po elements. The presynaptic elements contained numerous membrane-bound synaptic vesicles $v$, and both elements contained mitochondria $M$ and microtubules $\mathrm{m}$.

portions of the smooth endoplasmic reticulum and some golgi have swollen into electron transparent vacuoles, while the remainder of the cell showed no apparent alteration. This type of alteration was restricted to the neurons, and found only in tissuc irradiated at $289 \mathrm{~W} / \mathrm{cm}^{2}$. Other neurons exhibited the damage illustrated in Fig. 7(a), where both the cytoplasm and nucleoplasm exhibit reduced electron density, and the nuclei contain localized chromatin condensations. This was in contrast to the more homogeneous cytoplasmic and nucleoplasmic distributions observed in the control cells [Fig. 7(b)]. In these altered cells, portions of both the rough and smooth endoplasmic reticulum and the Golgi were swollen. Figure 8 shows a less commonly observed form of cellular damage. Aside from the abnormalities seen in Fig. 7(a), it should be noted how the subcellular organelles have pulled away from the cell periphery and moved towards the nucleus.

In those sections where tissue damage appeared in regions 3 and 5, altered morphology was both more pronounced and more extensively distributed throughout region 1. At the light microscope level, the tissue in region 1 appeared lighter staining and vacuolized (Fig. 9). The increasing degrees of ultrastructural damage observed in region 1 are illustrated in Fig. 10 with tissue taken from the locations indicated in Fig. 9(b). In location a [Fig. 10(a)] more mitochondria exhibited a greater degree of swelling, and the dendritelike processes also appeared more swollen and contained more membraneous debris. Some of the larger axons appeared swollen, and exhibited a reduction in the number of microtubules and neurofilaments. Figure 10(b) shows that the transparent vacuoles so prominent in location b [Fig. 9(b)] were swollen dendritelike processes. The large size of the swollen processes in this portion of the spinal cord may be attributed to their nearness to the cell bodies in region 5 , which may be their origin. The ringlike structures within the processes were most likely supra-swollen mitochondria whose outer membranes had ruptured, permitting the inner membrane to expand. This assumption is based on the observation of similar structures that were still partially confined by a second membrane. Frequently, tissue such as that from location c [Fig. 9(b)] was friable, and portions of it were lost from the spinal cord section during processing for electron microscopy. The electron microscope revealed [Fig. 10(c)] many electron transparent, membrane-bound regions devoid of internal structures, viz. the remnants of axons, axon bundles, and dendritelike processes.

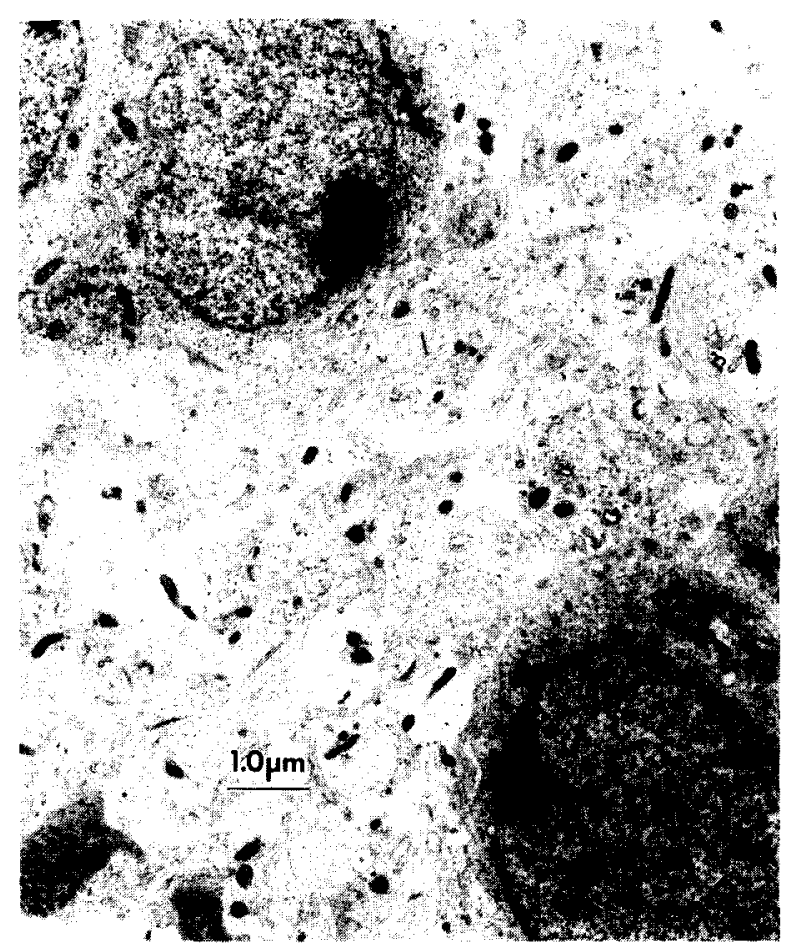

Fig. 4. Low magnification field of normal gray matter tissue. The most notable differences from the peripheral tissue were more numerous cell bodies and fewer nonmyelinated axons. 


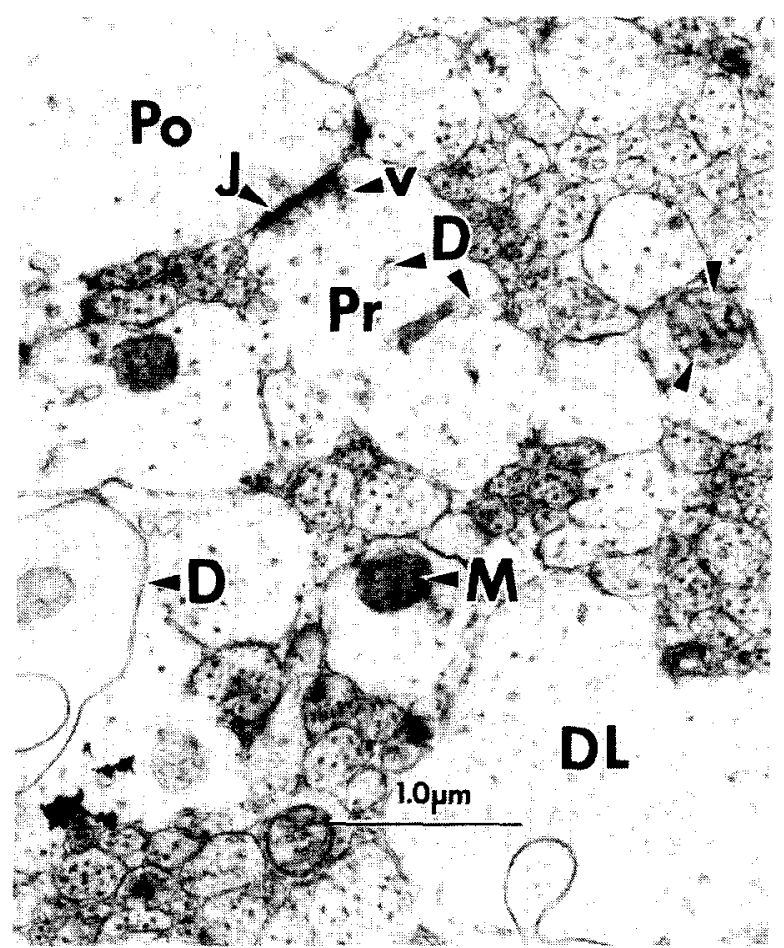

(a)

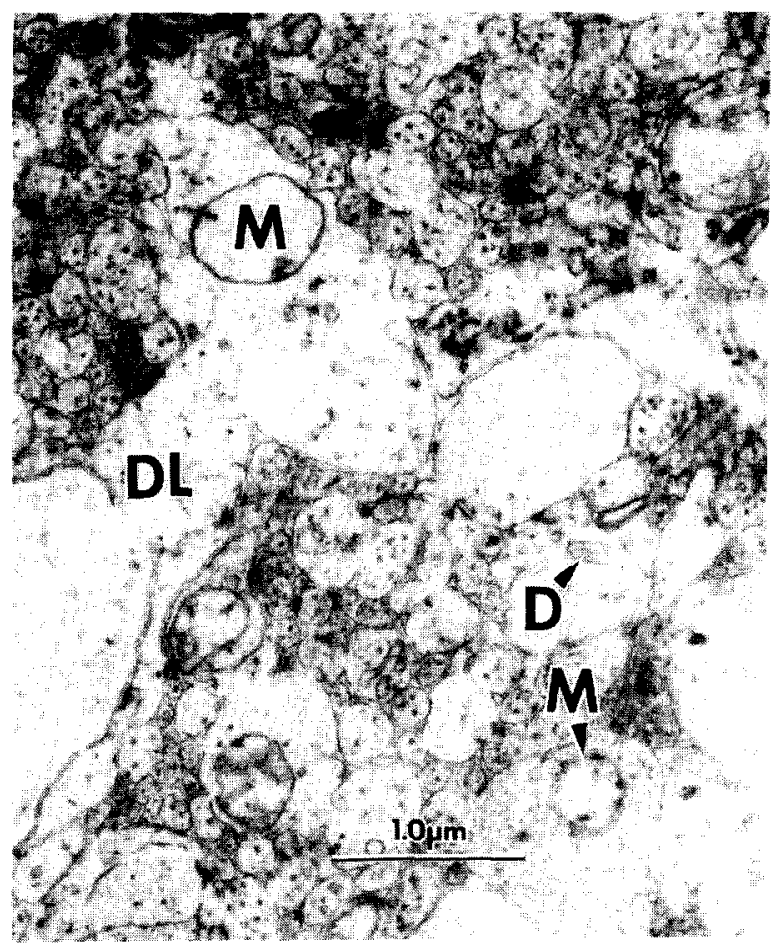

(b)

Fig. 5. Minimum tissuc damage associated with irreversible hind limb paralysis. The dendritelike processes DL, synapses, and cytoplasm appeared less electron dense and contained membranous debris D [(a) and (b)]. Most of the nonmyelinated axons appeared unaffected, although some did show an increase in their cytoplasm's electron density. Fewer synaptic vesicles $\mathrm{v}$ were observed in the presynaptic elements,

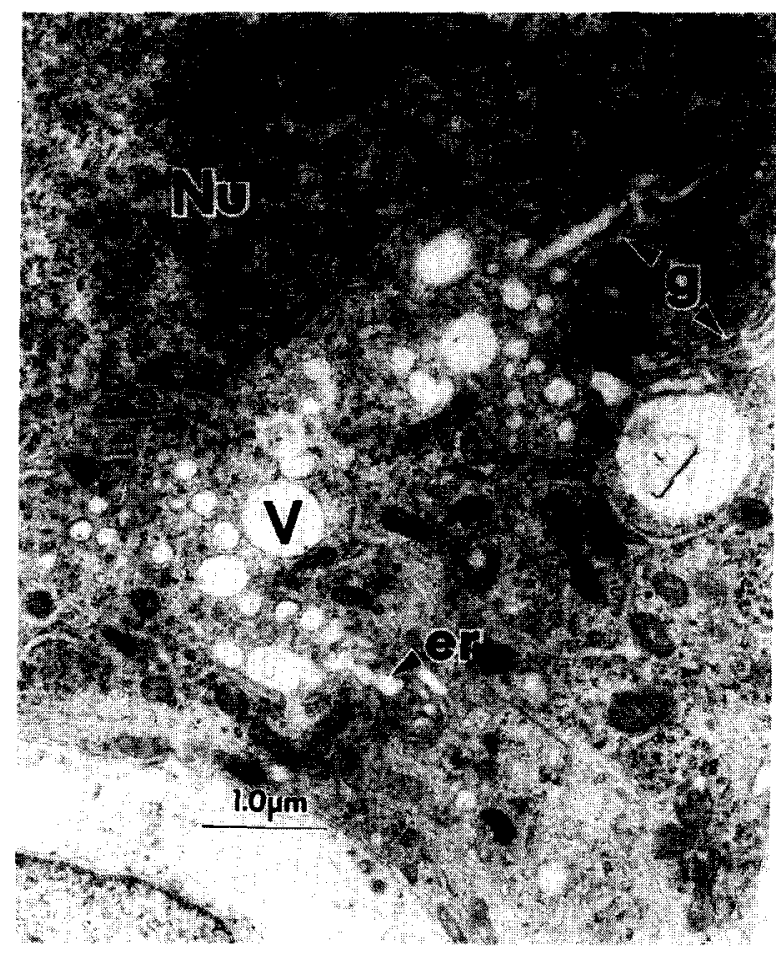

Fig. 6. Neuron in region 5 exhibiting vacuolization $V$ of the endoplasmic reticulum er and Golgi $\mathrm{g}$. This effect was observed only in tissue irradiated at $289 \mathrm{~W} / \mathrm{cm}^{2}$. No alterations were observed in the nuclei $\mathrm{Nu}$ of such cells.

In all specimens irradiated with $289 \mathrm{~W} / \mathrm{cm}^{2}$ at atmospheric pressure, tissue damage was restricted to the ventral half of the spinal cords, and did not extend into region 7 even when the damage in regions 1 , 3 , and 5 was clearly observable with light microscopy (Fig. 9). Mice irradiated at 16 atm hydrostatic pressure, with the corresponding increased $t_{90}$ exposure duration [Frizzell et al., 1983] or longer, exhibited tissue damage in rcgions 4 and 8 , and the acoustical monitor in the chamber showed a reduction in the half harmonic signal.

The effect of increasing post irradiation sacrifice time was to permit the development of a greater degree of tissue damage within the spinal cord regions exhibiting altered morphology immediately following the exposure. For example, Figs. 10(a)-(c) could represent temporal rather than spatial differences, such as would

and most of those remaining were aggregated at the now less distinct junctional densities $\mathrm{J}$ (a). The mitochondria $\mathrm{M}$ exhibited a wide range of altered states. Many had an overall reduction in matrix density; others had small electron transparent regions within their matrix [arrowheads in (a)]; while the most damaged mitochondria were simply electron transparent regions surrounded by a double membrane (b). 

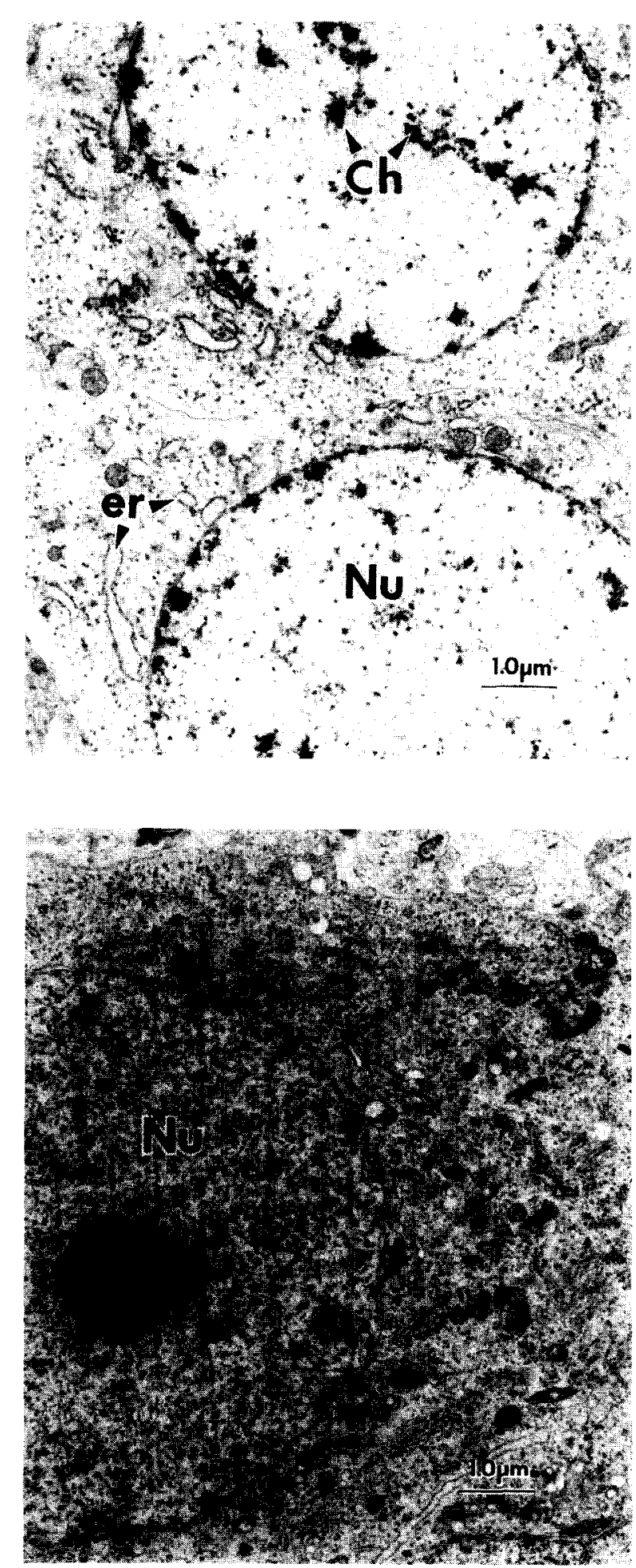

(b)

Fig. 7. Most altered neurons exhibited an electron density reduction of both their cytoplasm and nucleoplasm (a). The altered cells were characterized by condensed chromatin $\mathrm{Ch}$ in the nucleus $\mathrm{Nu}$ and swollen endoplasmic reticulum er (a). A normal neuron is shown for comparison (b). Note the prominent nucleolus $n$.

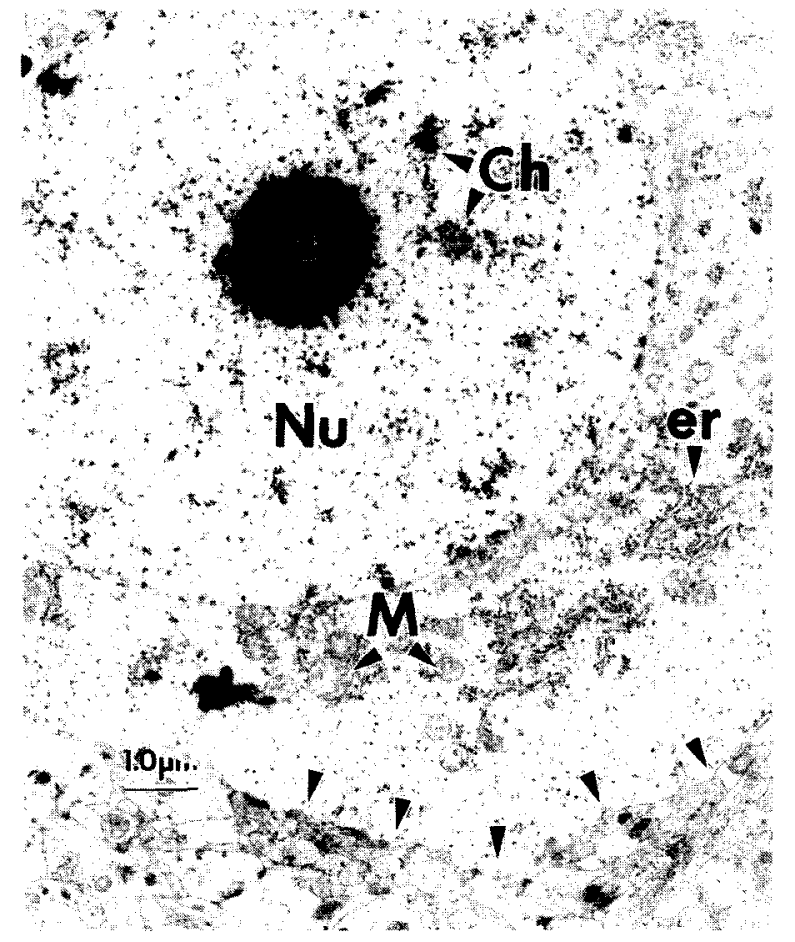

Fig. 8. A more rare form of altered neuron morphology. The damage is similar to that seen in Fig. 7(a), except that the subcellular organelles have pulled away from the cell periphery (arrowheads). Note that the nucleolus appears unaffected [compare to Fig. 7(b)].

be observed, $40 \mathrm{~min}, 2 \mathrm{~h}$, and $4 \mathrm{~h}$, respectively, following an exposure with initial damage similar to that depicted in Figs. 5(a) and (b).

\section{B. Altered morphology arising from exposure to inten- sities of $40-192 \mathrm{~W} / \mathrm{cm}^{2}$}

Except for the vacuolized endoplasmic reticulum depicted in Fig. 6, the nature of the tissue damage induced by the lower intensity exposures (40-192 W/ $\mathrm{cm}^{2}$ ) was the same as that described above for the corresponding exposure durations, i.e. $t_{10}, t_{50}$, and $t_{90}$. The observed difference was in the distribution of the tissue damage within the spinal cord sections. Damage remained greater in the peripheral tissue, but some of the $t_{50}$ and $t_{90}$ exposures induced damage in both the ventral and dorsal halves of the spinal cord (Fig. 11), though always to a grcater degree in the former. Those mice whose spinal cords exhibited a minimal level of damage (Fig. 5) in region 2 could not sense the stimulation. No damage was observed in this region in mice that recovered stimulation sensation.

Hyperbaric pressure did not alter the exposure duration thresholds [Frizzell et al., 1983] and had no apparent effect on the degree or distribution of tissue 


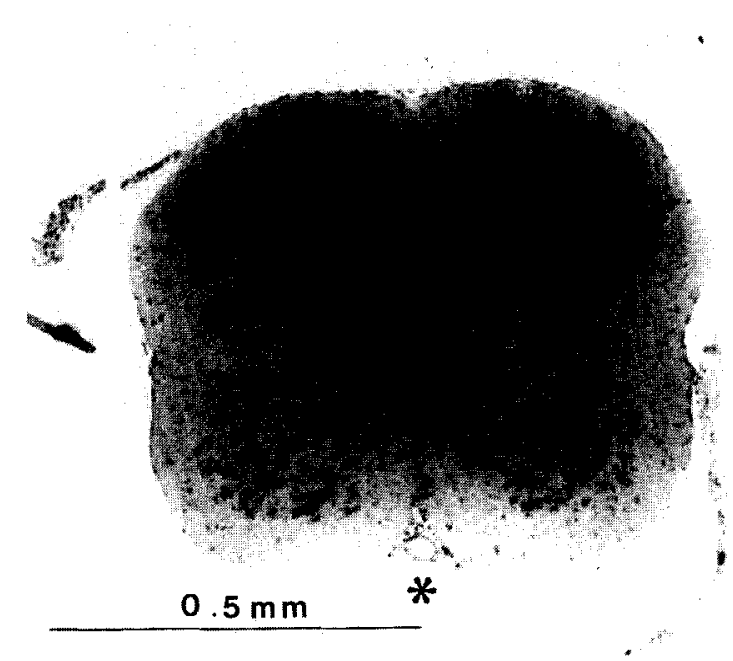

(a)

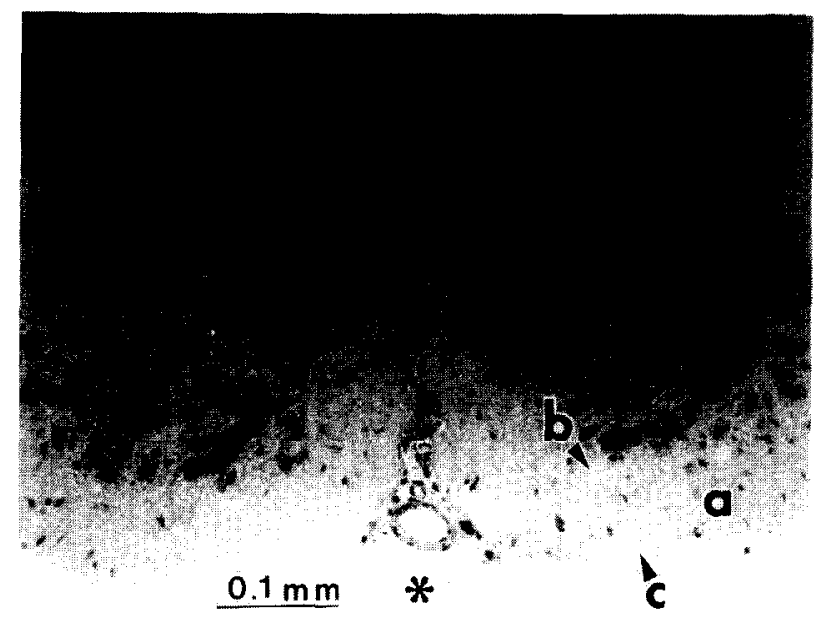

(b)

Fig. 9. Spinal cord section from mouse irradiated with a $t_{90}$ exposure at $289 \mathrm{~W} / \mathrm{cm}^{2}$ and sacrificed $15 \mathrm{~min}$ later. Tissue damage was restricted to the ventral half (asterisk) of the spinal cord (a). Compare this to Fig. 2(a). The higher magnification of (b) better illustrates the damage. The letters a, $\mathrm{b}$, and $\mathrm{c}$ in (b) indicate the regions from which the tissue was taken for, respectively, Figs. 10(a)-(c).

damage induced at intensities of $40-144 \mathrm{~W} / \mathrm{cm}^{2}$. The same was true for the $t_{90}$ exposure durations except at $86 \mathrm{~W} / \mathrm{cm}^{2}$, where $t_{90}$ increased by almost a factor of two.

\section{DISCUSSION}

For all exposure conditions employed, irreversible hind limb paralysis and stimulation sensation loss were always associated with altered morphology in the ventral and dorsal half of the spinal cord, respectively. The minimum level of tissue damage associated with these functional changes required the electron microscope for observation and was located at the spinal cord periphery where neuronal processes, viz. dendrites, axons, and synapses, are concentrated. It appears that damage to these structures interrupted neuronal transmissions. Hence, the neuron cell bodies themselves did not need to be affected by the ultrasound in order for the functions they governed to become impaired. There is always the possibility that it was altered morphology at the dorsal root or dorsal root ganglia that caused the stimulation sensation loss and damage in the ventral root that caused the hind limb paralysis. These structures were not examined.

It is interesting to observe that the synapses and dendritelike processes exhibited clearer indications of swelling and distortion than the surrounding axons. Also, the mitochondria in the axons appeared less affected by the ultrasonic exposure than were those in other structures. This could be accounted for by assuming that the axonal membranes differ significantly in their structural and/or permeability properties rendering them more resistant to ultrasonic irradiation than the synapses or dendritelike processes.

The distribution of altered spinal cord morphology associated with hind limb paralysis and stimulus sensation loss agrees with earlier findings [Brodal, 1969; Everett, 1971; Chusid, 1976]. Both the neurons and efferent axons associated with motor function are located in the ventral half of the spinal cord. Hence, tissue damage in the ventral half of the spinal cord would be expected to influence motor function. The case of the stimulus sensation loss is not so clear. Tissue damage in the dorsal half of the spinal cord could be expected to affect stimulus sensation since the associated afferent axons and neurons are located there. However, these nerve fibers cross over to the opposite half (left to right and vice versa) of the spinal cord and then ascend to the brain via pathways within the ventral half of the spinal cord. It would therefore be expected that damage in the ventral half of the spinal cord would also affect stimulus sensation. That such an effect was not observed may be explained by considering that nerve fibers need not cross over within the same spinal segment in which the neuron is located, but do so in adjacent segments. Some fibers may be altered in the ventral ascending pathways while others escape damage by crossing over and entering the ventral patnway in a spinal segment unaffected by the ultrasound.

Though the ultrasound was incident at the dorsal side, tissue damage was always greatest at the cord periphery, being more pronounced and extensive in the ventral than the dorsal half. It is suggested that the spinal column was responsible for this asymmetrical distribution of the tissue damage. It can be considered that the ultrasound attenuation of the column is greater 


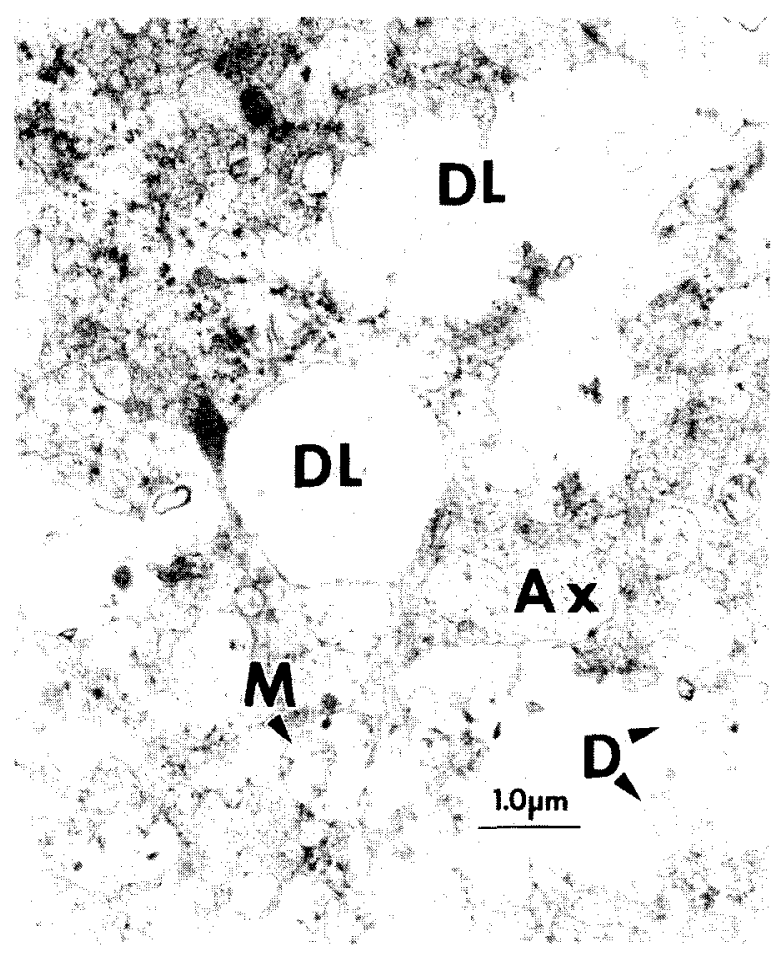

(a)

Fig. 10. Figures (a)-(c) represent, respectively, the damage characteristic of the regions marked a, b, and c in Fig. 9(b). These figures illustrate an increased degree of swelling in the axons Ax, dendritelike processes DL, and mitochondria $\mathbf{M}$ accompanied by a greater amount of membranous debris $\mathrm{D}$. The large size of the dendritelike processes in (b) may be because they were closer to their origin cells and had a greater girth than those in the peripheral tissue, even in the normal tissue. In (c), the tissue has a washed-out appearance, and even the axons show a loss of internal structure. Such tissue was frequently friable.

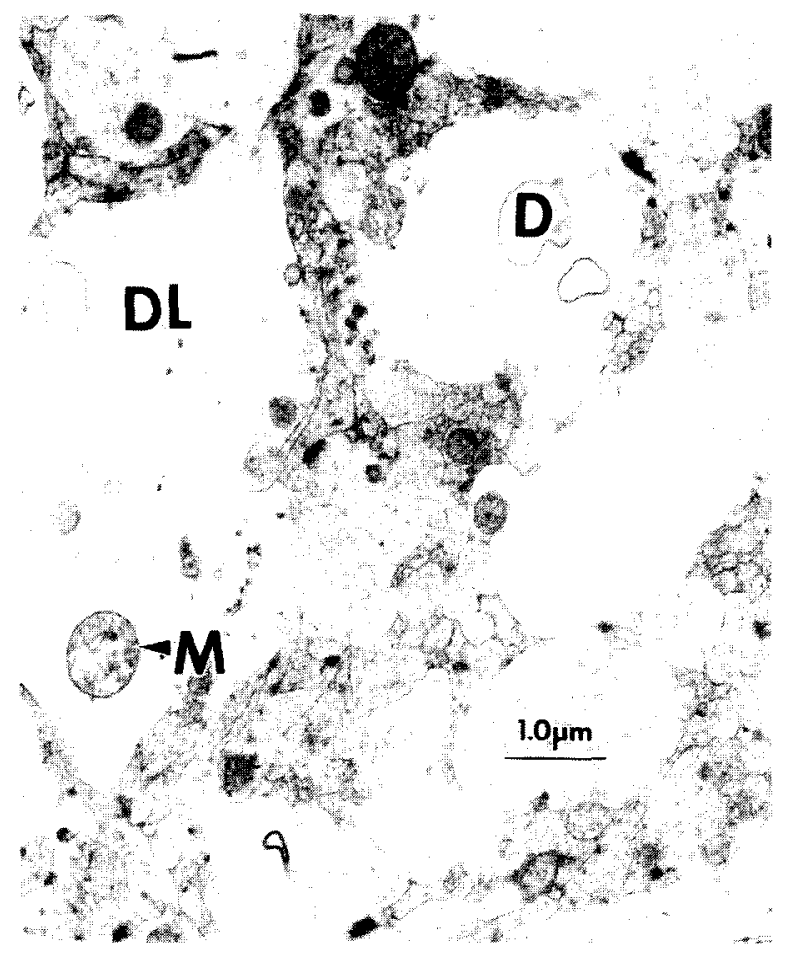

(b)

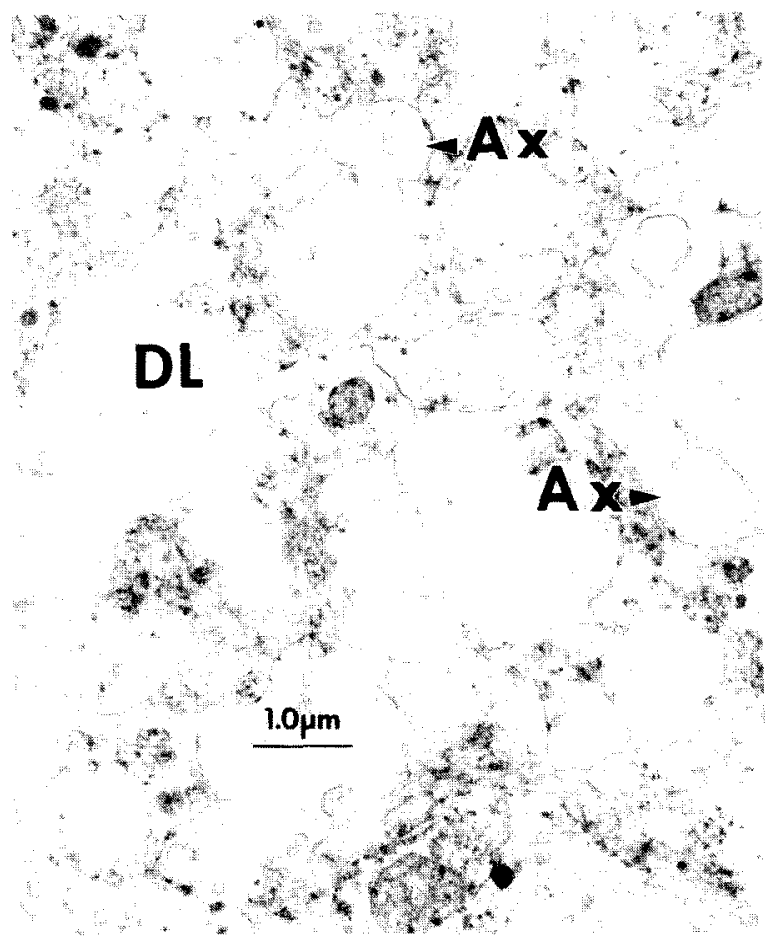

(c)

spinal cord at $1 \mathrm{MHz}$ [Dussik and Fritch, 1956; Goss et al., 1978]. The greater heat generated in the spinal column would in turn increase the temperature of the adjacent peripheral spinal cord tissue above that in the more centrally located tissues. This effect would be ex- (and presumably also is the absorption) than that of the spinal cord tissue. Although the spinal column of the mouse neonate contains more cartilage than bone [Fry and Dunn, 1956], the attenuation coefficient of cartilage is almost six times greater than that of the 
pected to be greater in the ventral half of the spinal cord as it is more massive (Fig. 12) and more ossified [Fry and Dunn, 1956]. Additionally, the spinal column, because of its significantly greater acoustic impedance, can be considered to affect the distribution of tissue damage in the cord by scattering and otherwise redirecting the acoustic field within the spinal cord. Focusing, the production of hot spots, etc., may occur as a result of the acoustic impedance mismatch and the complex geometry [Fry and Dunn, 1962].

The suggested influence of the spinal column on tissue damage distributions is based on the assumption that increased temperatures yielded more tissue damage either directly, i.e. damage arising from a thermal mechanism, or indirectly, i.e. the increased temperature enhanced the activity of a nonthermal mechanism. The increased threshold at $289 \mathrm{~W} / \mathrm{cm}^{2}$ under hyperbaric conditions together with the concomitant decrease of the half harmonic signal presumably emitted from bubbles suggests that acoustically induced cavitation was involved in producing the hind limb paralysis [Frizzell et al., 1983]. On acceptance of this, it may be speculated that cavitation was more intense in those tissue regions exhibiting the greatest damage. Damage was confined to the ventral portions of the spinal cord, for threshold exposures at $289 \mathrm{~W} / \mathrm{cm}^{2}$ and $1 \mathrm{~atm}$, but extended more toward the dorsal surface for threshold

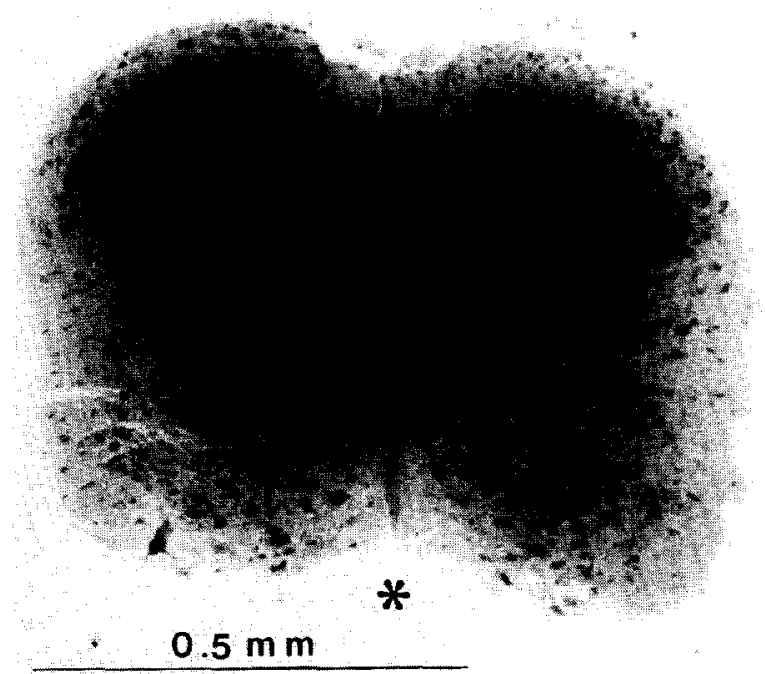

Fig. 11. Spinal cord section from mouse irradiated with a $t_{90}$ exposure at $144 \mathrm{~W} / \mathrm{cm}^{2}$ and sacrificed $15 \mathrm{~min}$ later. Note that damage is present in both the dorsal and ventral (asterisk) halves of the section.

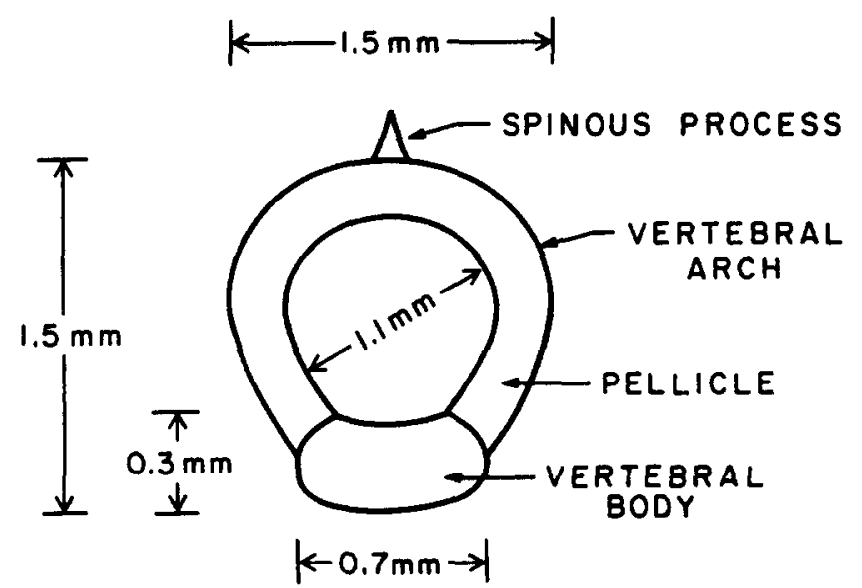

Fig. 12. Schematic drawing showing the dimensions of the spinal column and cord in the third lumbar vertebra.

exposures at $16 \mathrm{~atm}$, which is consistent with thermal enhancement of cavitation at the ventral surface at $1 \mathrm{~atm}$.

The threshold exposures for acoustic cavitation decrease with increasing gas content and with temperature of the medium [Coakley and Nyborg, 1978; Crum, 1982]. Hence, an increased temperature rise within the spinal cord tissue bordering the spinal column would create a more suitable environment for cavitation to occur. Since gas solubility decreases with increasing temperature, the free gas content in these regions would be higher, promoting the formation of cavitation nuclei or facilitating the growth of preexisting nuclei. There is evidence that acoustical phenomena can promote bubble production in vivo [ter Haar et al., 1982].

The damage observed is not similar to other known cavitation induced damage wherein transient events were believed to produce catastrophic events [Fry et al., 1970]. Another mechanism which may be considered is microstreaming in the neighborhood of oscillating bubbles [El'piner et al., 1965; Nyborg, 1968]. This has been well demonstrated in in vitro cell suspensions, though demonstration has not yet been made to show that such events can occur in vivo. It is also possible that increased loss and increased heat generation associated with bubbles produced the changes observed.

Cavitation may also induce chemical changes in the cell suspensions, such as the formation of free radicals [Flynn, 1964]. The addition of radical scavengers to ultrasonically irradiated cells in suspension protected against cell killing [Fu et al., 1979], particularly radicals capable of crossing the cell membrane [Armour and Corry, 1982]. The fact that a membrane crossing radical scavenger provided better protection suggests that radicals were formed within the cell. 
At those intensities where the threshold was unaffected by the hyperbaric pressure, the tissue damage is considered not to be produced by cavitation events. Rather, tissue damage is considered to have been produced by the ultrasonically induced hyperthermia or other mechanisms intimately related to the physical properties of the tissue, such as ultrasonic hysteresis [Johnston and Dunn, 1981].

Acknowledgment-This work was supported in part by grants from the National Institutes of Health.

The authors would like to thank the staff of the University of Illinois Center for Electron Microscopy, particularly Dr Birute Jakstys, Dr Richard Crang, Dr Marcella Gillott, and Mr Richard Olsen, for suggestions on specimen preparation and interpretation of the micrographs.

\section{REFERENCES}

Armour E. P. and Corry P. M. (1982) Cytotoxic effects of ultrasound: in vitro dependence on gas content, frequency, radical scavengers, and attachment. Radiat. Res. 89, 369-380.

Borrelli M. J., Bailey K. I. and Dunn F. (1981) Early ultrasonic effects upon mammalian CNS structures (chemical synapses). J. Acoust. Soc. Am. 69, 1514-1516.

Brodal A. (1969) Neurological Anatomy. Oxford University Press, New York.

Chusid J. G. (1976) Correlative Neuroanatomy and Functional Neurology. Lange Medical Publications, Los Altos, CA.

Coakley W. T. and Nyborg W. L. (1978) Cavitation; dynamics of gas bubbles; applications. In Ultrasound; Its Applications in Medicine and Biology (Edited by F. J. Fry), pp. 77-159. Elsevier, Amsterdam.

Crum L. A. (1982) Nucleation and stabilization of microbubbles in liquids. Appl. Sci. Res. 37, 101-115.

Duffy C. J. and Tyler T. J. (1975) A simple tissue slicer. Physiology and Behavior 14, 525-526.

Dunn F. (1958) Physical mechanisms of the action of intense ultrasound on tissue. Am. J. Phys. Med. 37, 148-151.

Dussik K. T. and Fritch D. J. (1956) Determination of sound attenuation and sound velocity in the structure constituting the joints, and of the ultrasonic field distribution within the joints on the living tissues and anatomical preparations, both in normal and pathological conditions. Public Health Service, Natl. Inst. Health Project A454, Progr. Rep. (15 September 1956).

El'Piner I. E., Faiken I. M. and Basurmanova O. K. (1956) Intracellular microcurrents caused by ultrasound waves. Biofizika 10, 805-809.

Everett N. B. (1971) Functional Neurology. Lea and Febiger, Philadelphia.

Flynn H. G. (1964) Physics of acoustic cavitation in liquids. In Physical Acoustics (Edited by W. P. Mason), Chap. 9, Vol. I, Part B, pp. 57-172. Academic, New York.

Frizzell L. A., Lee C. S., Aschenbach P. D., Borrelli M. J., Morimoto R. S. and Dunn F. (1983) Involvement of ultrasonically induced cavitation in hind limb paralysis of the mouse neonate. J. Acoust. Soc. Am. 74, 1062-1065.

Fry F. J., Kossoff G., Eggleton R. C. and Dunn F. (1970) Threshold ultrasonic dosages for structural changes in mammalian brain. $J$. Acoust. Soc. Am. 48, 1413-1417.

Fry W. J. and Dunn F. (1956) Ultrasound irradiation of the central nervous system at high sound levels. J. Acoust. Soc. Am. 28, 129 131.

Fry W. J. and Dunn F. (1962) Ultrasound: Analysis and Experimental Methods in Biological Research. In Physical Techniques in Biological Research (Edited by W. L. Nastuk). Vol. IV, Chap. 6, pp. 261-394. Academic, New York.

Fu Y. K., Kaufman G. E., Miller M. W., Griffiths T. D. and Lange C. S. (1979) Modification by cysteamine of ultrasound lethality to chinese hamster V-79 cells. Radiat. Res. 80, 575-580.

Goss S. A., Johnston R. L. and Dunn F. (1978) Comprehensive compilation of empirical properties of mammalian tissues. $J$. Acoust. Soc. Am. 64, 423-457.

Johnston R. L. and Dunn F. (1981) Ultrasonic hysteresis in biological media. Radiat. Env. Biophys. 19, 137-148.

Nyborg W. L. (1968) Mechanisms for nonthermal effects of ultrasound. J. Acoust. Soc. Am. 44, 1302-1309.

Rugh R. (ed) (1968) The Mouse: Its reproduction and development. Burgess, Minneapolis.

Taylor K. J. W. (1970) Ultrasonic damage to spinal cord and the synergistic effect of hypoxia. J. Pathol. 102, 41-47.

ter Haar G., Daniels S., Eastaugh K. C. and Hill, C. R. (1982) Ultrasonically induced cavitation in vivo. Br. J. Cancer 45, Suppl. V, 15I-155. 